\title{
Supporting Information for: A Pictorial View of Viscosity in Ionic Liquids and the Link to Nanostructural Heterogeneity
}

\author{
Weththasinghage D. Amith ${ }^{\dagger}$ Juan C. Araque,$^{\ddagger}$ and Claudio J. Margulis ${ }^{*, \dagger}$ \\ $\dagger$ Department of Chemistry, University of Iowa, Iowa City, Iowa 52242, United States \\ $\ddagger$ School of Engineering, Benedictine College, Atchison, Kansas 66002, United States \\ E-mail: claudio-margulis@uiowa.edu
}


For $\mathrm{Im}_{1,8}^{+} / \mathrm{NTf}_{2}^{-}$in the bulk phase, the 10 ns constant energy (NVE) molecular dynamics trajectory from which $S(q, t)$ was derived is the same as that used in a recent study ${ }^{1}$ and details regarding equilibration are provided there. As described in our prior work, force-field parameters were obtained from the Canongia-Lopes and Pádua ${ }^{213}$ and optimized potentials for liquid simulations all-atoms (OPLS-AA) ${ }^{4}$ force fields inlcuding the Köddermann et. al. modifications to Lennard Jones parameters. ${ }^{5}$ The final configuration of the NVE production run was used as input for further simulations in a series of independent constant temperature (NVT) runs from which the time-dependent stress tensor correlation function from which the viscosity is derived was computed using the protocol proposed by the Maginn group.$^{6}$ These NVT trajectories were generated using the GROMACS (4.5.5-double precision) package. $\frac{78}{}$ To properly average and converge the time dependent viscosity, 65 independent NVT trajectories ( 7 ns each run) were generated from different velocity seeds. The temperature for the NVT ensemble was considered as the average temperature from the NVE production run which was $377.403 \mathrm{~K}$. All NVT simulations used the velocity-Verlet ${ }^{9}$ MD integrator and the Nose-Hoover $\frac{10111}{1}$ thermostat with a 0.5 ps time constant. Long-range Coulomb interactions were handled using the Particle-Mesh Ewald method ${ }^{12113}$ with a $0.08 \mathrm{~nm}$ Fourier spacing and a sixth order interpolation. The cutoff for all the non-bonded interactions was considered to be $1.5 \mathrm{~nm}$.

The calculation of $S(q, t)$ goes along the same lines as that of $S(q)^{14115}$ except that instead of using the radial distribution function $g_{i j}(r)$ between atom types $i$ and $j$, we use the distinct van Hove correlation function $G_{d}^{i j}(r, t)$.

$$
S(q, t)=\frac{\rho_{0} \sum_{i} \sum_{j} x_{i} x_{j} f_{i}(q) f_{j}(q) \int_{0}^{L / 2} 4 \pi r^{2}\left(g_{d}^{i j}(r, t)-1\right)\left(\frac{\sin q r}{q r}\right) W(r) d r}{\left[\sum_{i} x_{i} f_{i}(q)\right]^{2}},
$$

where

$$
g_{d}^{i j}(r, t)=\frac{G_{d}^{i j}(r, t)}{\rho} .
$$

$G_{d}^{i j}(r, t)$ was computed using an in-house modified version of the LiquidLib toolbox. ${ }^{16}$ 
In Eq. S.1, $\rho_{0}$ is the total number density of the system, $x_{i}$ and $x_{j}$ are the fractions of atoms of type $i$ and $j, f_{i}(q)$ and $f_{j}(q)$ are the X-ray atomic form factors and $W(r)$ is a Lorch function. $\frac{14|15| 17 \mid 18}{\rho} \rho$ in the Eq. $\mathrm{S.2}$ is the long distance limit of $G_{d}^{i j}(r, t)$ such that $G_{d}^{i j}(r, t) / \rho$ goes to one at long $r . \underline{16}$ In the main manuscript, we put emphasis on the partial subcomponent $S^{H-A}(q, t)$ corresponding specifically to cationic head-anion correlations. For the purpose of the calculation of the dynamic structure function and its subcomponents, the definition of cationic head includes all atoms in the imidazolium ring, the methyl group and atoms up to the second carbon in the octyl tail. The rest of the octyl tail is considered the apolar subcomponent.

To estimate the time constants for decorrelation of $S^{H-A}(q, t)^{2}$ at different $q$ values we fitted these function (functions and fits shown in Figs. S.1, S.2 and S.3) using single and double exponential expressions as in Eqns. S.3 and S.4. Time constants derived from the fits are provided in table S.1.

$$
\begin{gathered}
f(t)=N e^{-t / \tau} \\
f(t)=N_{0} e^{-t / \tau_{1}}+N_{1} e^{-t / \tau_{2}}
\end{gathered}
$$

Table S.1: Time Constants for Eqns. S.3 and S.4.

\begin{tabular}{|c|c|c|}
\hline \multicolumn{3}{|c|}{ Time Constant $(\mathrm{ps})$} \\
\hline Range & $\tau$ & $\tau_{1}, \tau_{2}$ \\
\hline q $_{\text {polarity }}$ & 370.37 & $94.340,416.67$ \\
q charge & 63.694 & $6.9784,75.758$ \\
qadjacency & 10.194 & $2.0500,19.455$ \\
\hline
\end{tabular}

The calculation of viscosity followed closely the protocol in reference 6 . The cutoff time $\left(\mathrm{t}_{\text {cut }}\right.$ ) beyond which $\mu$ was considered converged was set to 984 ps. For $q=q_{\text {adjacency }}$ and $q=q_{\text {charge }}$ this time was also used to normalize the integral of $S^{H-A}(q, t)^{2}$ in Figure 3 of 


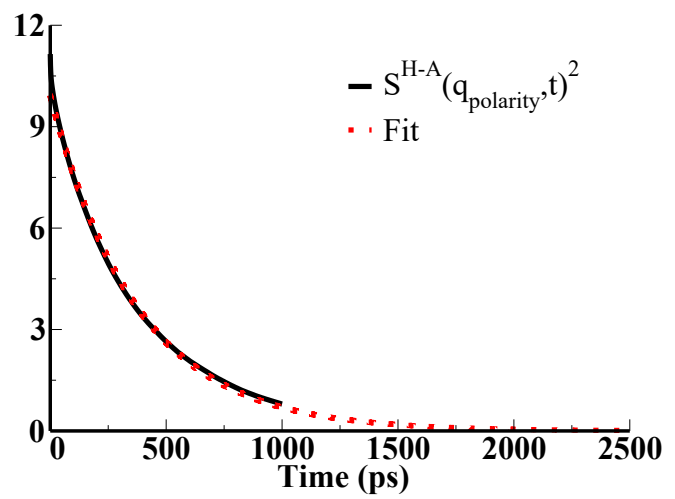

(a)

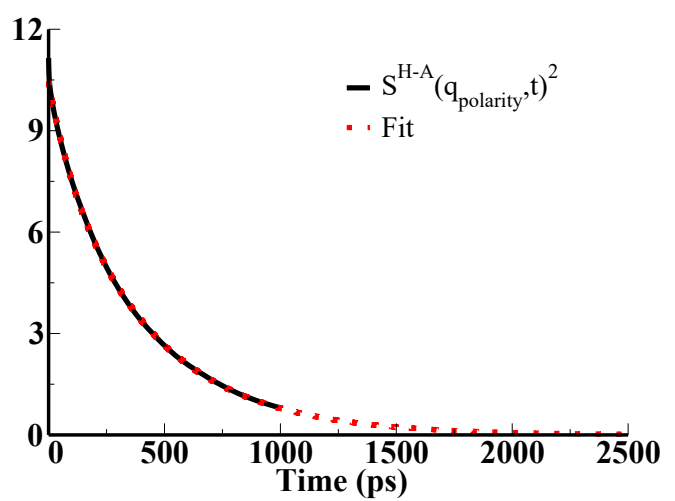

(b)

Figure S.1: Single exponential (a) and double exponential (b) fits of $S^{H-A}\left(q_{\text {polarity }}, t\right)^{2}$.

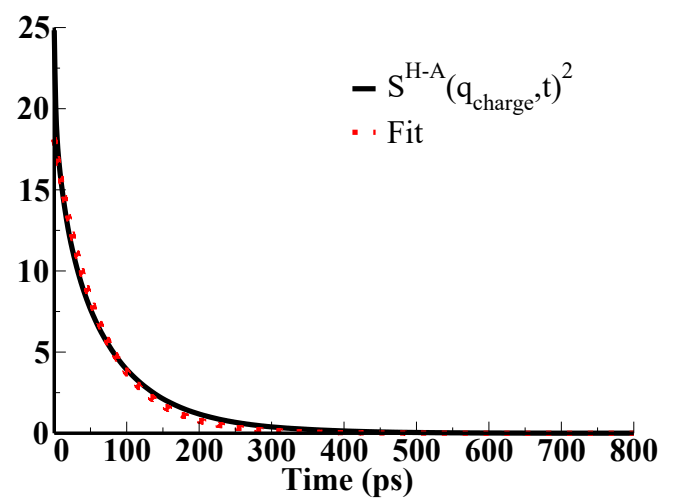

(a)

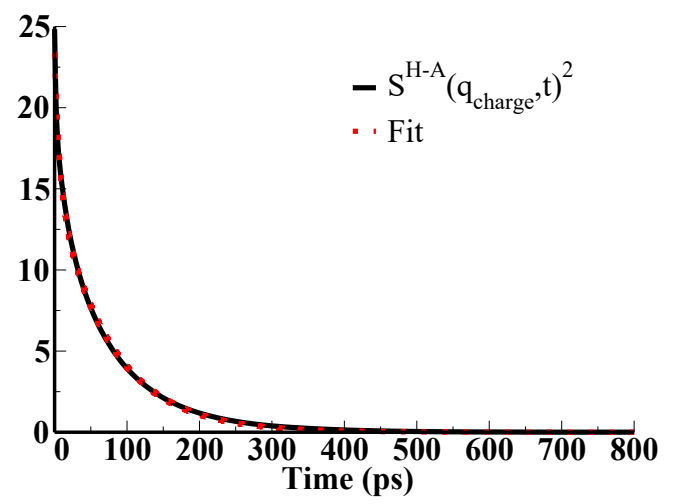

(b)

Figure S.2: Single exponential (a) and double exponential (b) fits of $S^{H-A}\left(q_{\text {charge }}, t\right)^{2}$. 


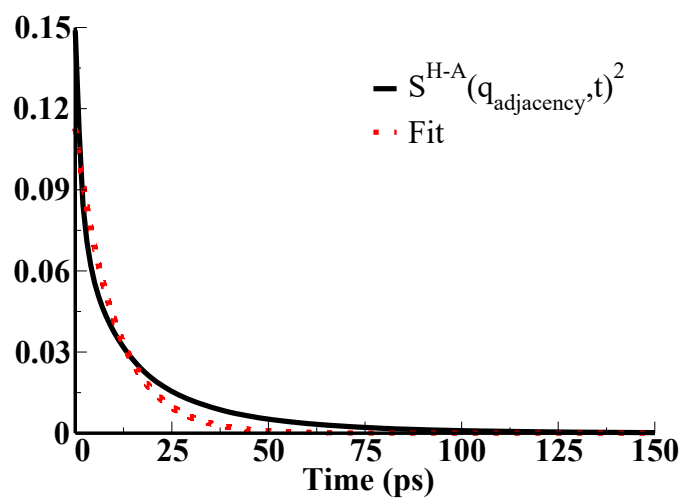

(a)

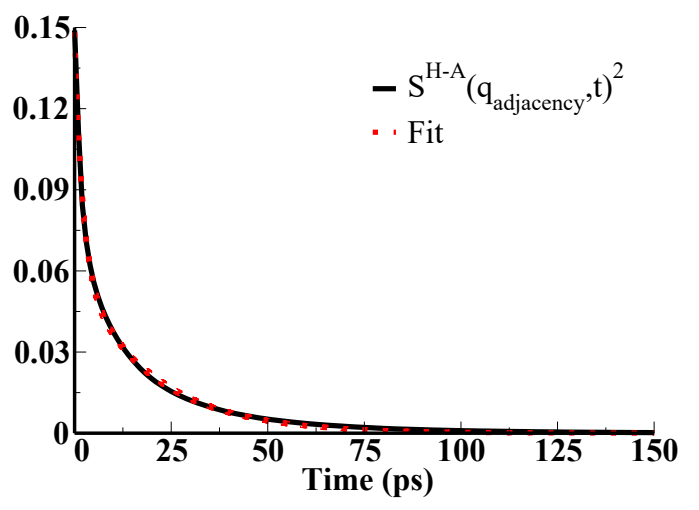

(b)

Figure S.3: Single exponential (a) and double exponential (b) fits of $S^{H-A}\left(q_{\text {adjacency }}, t\right)^{2}$.

the main text. For $q_{\text {polarity }}$, the time scale for full decay of correlations in $S^{H-A}(q, t)^{2}$ was significantly beyond $984 \mathrm{ps}$ as can be seen from Fig S.1. To generate the curve at $q_{\text {polarity }}$ in Fig. 3 of the main text, we integrated the double exponential fit and normalized this integral at $\mathrm{t}_{\text {cut }}=2500 \mathrm{ps}$, beyond which the integrand is for all practical purposes zero.

In Fig. 4 of the main text, the surfaces represent an isovalue of the concentration profile of polar (a through e) and apolar (e) subcomponents. For any atomic selection of a system subcomponent $i$, its concentration (or density) profile $\rho_{i}(\mathbf{r})$ was calculated according to the method developed by Schultz et al., $\frac{1920}{20}$ where structural information is extracted from the unweighted (i.e. without form factors) subcomponent structure factor $\tilde{S}_{i}(\mathbf{q})$ obtained from molecular dynamics trajectories (see references 19 and 20 for more details). $\rho_{i}(\mathbf{r})$ in the simulation box is calculated as a Fourier expansion, 20

$$
\rho_{i}(\mathbf{r})=\rho_{i}\left(1+\sum_{k} \beta_{i, k} \sin \left(\mathbf{q}_{k} \cdot \mathbf{r}+\theta_{i, k}\right)\right)
$$

where $\beta_{i, k}, \theta_{i, k}$ are the subcomponent amplitude and phase angle at wavevector $\mathbf{q}_{k}$ defined $\operatorname{as}^{20}$

$$
\beta_{i, k}=\frac{2}{N_{i}} \sqrt{\tilde{S}_{i}\left(\mathbf{q}_{k}\right) N}
$$


and

$$
\theta_{i, k}=\tan ^{-1}\left(\frac{\sum_{i_{\text {atom }}} \cos \left(\mathbf{q}_{k} \cdot \mathbf{r}_{i_{\text {atom }}}\right)}{\sum_{i_{\text {atom }}} \sin \left(\mathbf{q}_{k} \cdot \mathbf{r}_{i_{\text {atom }}}\right)}\right)
$$

respectively; $\rho_{i}$ is the subcomponent number density. $N_{i}$ is the number of atoms belonging to subcomponent $i$ and $N$ is the total number of atoms in the system. $\frac{1920}{20}$ Sums in equation S.7 are done exclusively for atoms in subcomponent $i\left(i_{\text {atom }}=1\right.$ through $\left.N_{i}\right)$; in other words, these have a weighting factor of one, whereas the factor is zero for all other atoms in the system. A wavevector cutoff of $\left|\mathbf{q}_{k}\right|<2 \AA^{-1}$ was used in this work in order to reduce the noise in the real space concentration profile. The simulation box was divided into cubic voxels (either $50 \times 50 \times 50$ or $100 \times 100 \times 100$ ) and concentration profiles were obtained at the geometric center of each voxel. Schultz argues $\frac{19[20}{20}$ that isosurfaces at isovalues $\rho_{i}(\mathbf{r}) / \rho \geq 0.5$ appropriately enclose atoms in the subcomponent of interest and in Fig. 4, an isovalue of 0.82 was used because it provides aesthetically pleasing isosurfaces; here $\rho$ is the overall number density.

For the purpose of coloring the previously defined polar isosurface, we also needed independent cationic head and anionic density profiles which were computed and voxels given a value different from zero if $\rho_{i}(\mathbf{r}) / \rho \geq 0.5$; cationic heads included all atoms up to the first $-\mathrm{CH}_{2^{-}}\left(-\mathrm{CH}_{3}\right)$ group attached to imidazolium rings. The anionic density profile was multiplied by -1 so that positive values were used to color the polar isosurface when it coincides with significant cationic number density and negative values when it coincides with significant anionic number density; with this criteria, the coloring of the polar isosurface in Fig. 4a is red when at the location of this surface, voxels are majoritarily anionic and blue when majoritarily cationic-head. The color scale is setup so that red is represented by a voxel value of -1.0 (or more negative), blue by +1.0 (or more positive) and white by 0.0 ; values in between get an interpolated color. At later times, the colors are obtained as equal weight linear combinations of the current time voxel and the zero time voxel and always evaluated at the location of the zero time polar network. For polar and apolar linear combinations in Fig. 4d a similar protocol is used. 


\section{References}

(1) Araque, J. C.; Margulis, C. J. In an ionic liquid, high local friction is determined by the proximity to the charge network. The Journal of Chemical Physics 2018, 149, 144503.

(2) Lopes, J. N. C.; Deschamps, J.; Pádua, A. A. H. Modeling Ionic Liquids Using a Systematic All-Atom Force Field. The Journal of Physical Chemistry B 2004, 108, $2038-2047$.

(3) Lopes, J. N. C.; Pádua, A. A. H. Molecular Force Field for Ionic Liquids Composed of Triflate or Bistriflylimide Anions. The Journal of Physical Chemistry B 2004, 108, $16893-16898$.

(4) Jorgensen, W. L.; Maxwell, D. S.; Tirado-Rives, J. Development and Testing of the OPLS All-Atom Force Field on Conformational Energetics and Properties of Organic Liquids. Journal of the American Chemical Society 1996, 118, 11225-11236.

(5) Köddermann, T.; Paschek, D.; Ludwig, R. Molecular Dynamic Simulations of Ionic Liquids: A Reliable Description of Structure, Thermodynamics and Dynamics. ChemPhysChem 2007, 8, 2464-2470.

(6) Zhang, Y.; Otani, A.; Maginn, E. J. Reliable Viscosity Calculation from Equilibrium Molecular Dynamics Simulations: A Time Decomposition Method. Journal of Chemical Theory and Computation 2015, 11,3537-3546.

(7) Hess, B.; Kutzner, C.; van der Spoel, D.; Lindahl, E. GROMACS 4: Algorithms for Highly Efficient, Load-Balanced, and Scalable Molecular Simulation. J. Chem. Theory Comput. 2008, 4, 435-447.

(8) Van Der Spoel, D.; Lindahl, E.; Hess, B.; Groenhof, G.; Mark, A. E.; Berendsen, H. J. C. GROMACS: Fast, Flexible, and Free. J. Comput. Chem. 2005, 26, 1701-1718. 
(9) Verlet, L. Computer "Experiments" on Classical Fluids. I. Thermodynamical Properties of Lennard-Jones Molecules. Physical Review 1967, 159, 98-103.

(10) Nosé, S. A Unified Formulation of the Constant Temperature Molecular Dynamics Methods. The Journal of Chemical Physics 1984, 81, 511-519.

(11) Nosé, S. A molecular dynamics method for simulations in the canonical ensemble. Molecular Physics 1984, 52, 255-268.

(12) Darden, T.; York, D.; Pedersen, L. Particle Mesh Ewald: An N $\log (\mathrm{N})$ Method for Ewald Sums in Large Systems. The Journal of Chemical Physics 1993, 98, 1008910092.

(13) Essmann, U.; Perera, L.; Berkowitz, M. L.; Darden, T.; Lee, H.; Pedersen, L. G. A Smooth Particle Mesh Ewald Method. The Journal of Chemical Physics 1995, 103, $8577-8593$.

(14) Araque, J. C.; Hettige, J. J.; Margulis, C. J. Modern Room Temperature Ionic Liquids, a Simple Guide to Understanding Their Structure and How It May Relate to Dynamics. The Journal of Physical Chemistry B 2015, 119, 12727-12740.

(15) Kashyap, H. K.; Margulis, C. J. (Keynote) Theoretical Deconstruction of the X-ray Structure Function Exposes Polarity Alternations in Room Temperature Ionic Liquids. ECS Transactions 2013, 50, 301-307.

(16) Walter, N. P.; Jaiswal, A.; Cai, Z.; Zhang, Y. LiquidLib: A comprehensive toolbox for analyzing classical and ab initio molecular dynamics simulations of liquids and liquidlike matter with applications to neutron scattering experiments. Computer Physics Communications 2018, 228, 209-218.

(17) Lorch, E. Neutron diffraction by germania, silica and radiation-damaged silica glasses. Journal of Physics C: Solid State Physics 1969, 2, 229-237. 
(18) Du, J.; Benmore, C. J.; Corrales, R.; Hart, R. T.; Weber, J. K. R. A molecular dynamics simulation interpretation of neutron and x-ray diffraction measurements on single phase $\mathrm{Y}_{2} \mathrm{O}_{3}-\mathrm{Al}_{2} \mathrm{O}_{3}$ glasses. Journal of Physics: Condensed Matter 2009, 21, 205102.

(19) Schultz, A. J. Modeling and Computer Simulation of Block Copolymer/Nanoparticle Composites. Ph.D. thesis, North Carolina State University, Raleigh, NC 27695, 2004.

(20) Schultz, A. J.; Hall, C. K.; Genzer, J. Obtaining Concentration Profiles from Computer Simulation Structure Factors. Macromolecules 2007, 40, 2629-2632. 